\title{
Effects of Proprioceptive Neuromuscular Facilitation Neck Flexion Exercise and the Shaker Exercise on the Activities of the Suprahyoid Muscles in Chronic Stroke Patients with Dysphagia
}

\author{
Gak Hwangbo, PT, PhD - Kyoung-Don Kim, PT, $\mathrm{PhD}^{1 \dagger}$ \\ Department of Physical Therapy, Daegu University \\ ${ }^{1}$ Department of Physical Therapy, Dongju College
}

Received: July 25, 2018 / Revised: July 27, 2018 / Accepted: October 4, 2018

(C) 2018 J Korean Soc Phys Med

\section{| Abstract |}

PURPOSE: The purpose of this study is to examine the effect of proprioceptive neuromuscular facilitation (PNF) neck flexion exercise and the Shaker exercises on the activity of the suprahyoid muscles in chronic stroke patients with dysphagia and to show what exercise methods are effective for swallowing rehabilitation.

METHODS: This study was conducted at F hospital in Daegu from August 2014 to February 2017 with 60 participants who understood the purpose of the study and desired to participate. The 60 participants were randomly divided into an experimental group (PNF neck flexion exercise) $(n=30)$ and a control group (Shaker exercise) $(n=30)$. PNF neck flexion exercise was performed in the experimental group and the Shaker exercise was performed in the control group for 30 minutes, 5 times a week for 6 weeks.

†Corresponding Author : Kyoung-Don Kim doniee1595@gmail.com, http://orcid.org/0000-0001-7173-2004 This is an Open Access article distributed under the terms of the Creative Commons Attribution Non-Commercial License (http://creativecommons.org/licenses/by-nc/3.0) which permits unrestricted non-commercial use, distribution, and reproduction in any medium, provided the original work is properly cited.
The activity of the suprahyoid muscles was measured before and after treatment.

RESULTS: Both the experimental group and the control group showed a statistically significant change in the activity of the suprahyoid muscles before and after the treatment. The changes were also statistically significant when compared by group.

CONCLUSION: In conclusion, PNF neck flexion exercise should be used in addition to Shaker exercise when rehabilitating a patient with a swallowing disability.

Key Words: Dysphagia, Stroke, Swallowing

\section{Introduction}

Patients with dysphagia need to improve nutritional intake, decrease morbidity and mortality associated with chest infections due to airway aspiration, and resume their normal diet from prior to the onset of dysphagia (Singh and Hamdy, 2006). The swallowing process consists of the oral preparatory phase, the oral phase, the pharyngeal phase, and the esophageal phase (Kim, 2015). One method of alleviating dysphagia in the pharyngeal phase is to enhance the activity of muscles involved in swallowing 
(Woo et al., 2012). The suprahyoid muscles are very important for safe swallowing (Oh, 2018). When the suprahyoid muscles are strengthened, the upper esophageal sphincter opens well, which reduces the risk of inhalation by reducing the amount of food residue after swallowing (Shaker et al., 1997). One of the most common methods to strengthen musculi suprahyoidei is the Shaker exercise. The Shaker exercise is an isotonic and isometric exercise that involves lifting the head while lying down. Shaker et al. (2002) reported that for patients with oropharyngeal swallowing difficulties, the Shaker exercise is effective to strengthen the musculi suprahyoidei and to open the upper esophageal sphincter. Logemann et al. (2009) also reported that the Shaker exercise helps to reduce aspiration. Indeed, many previous studies have reported that the Shaker exercise is helpful with swallowing. (Easterling et al., 2000; Shaker et al., 2002; White et al., 2008; Logemann et al., 2009; Mepani et al., 2009; Hong et al., 2012; Choi et al., 2017).

However, there are various disadvantages to the Shaker exercise. Easterling et al. (2000) had 26 seniors conduct the Shaker exercise for six weeks. As a result, 24 of them reported neck pain, dizziness and discomfort upon exercise while 14 left in the middle due to difficulties of continued supervision and guidance. Also, in order to perform the Shaker exercise, neck bending muscular strength must be fair or above (Kim, 2015). Given these disadvantages, other exercises can be used to strengthen musculi suprahyoidei.

Another exercise to strengthen musculi suprahyoidei is proprioceptive neuromuscular facilitation (PNF) neck flexion exercise. PNF will stimulate proprioception, promote workings of the muscles and nerves, and enable a functional approach. It is applied to stroke and various other diseases (Adler et al., 2000). PNF patterns also induce muscular contraction in diagonal and spiral directions and can be applied to sports and other areas (Kofotolis and Kellis, 2006). Lee and Won (2015) reported in their case study that PNF-based neck muscle strengthening exercise will reduce aspiration and improve swallowing function of patients with a swallowing disability.

Compared with the Shaker exercise, there are very few studies on the effect of PNF neck flexion exercise on the muscular activity of musculi suprahyoidei. According to Kim (2015), both PNF neck bending and the Shaker exercise effectively strengthen the suprahyoid muscles in patients with dysphagia, but additional research is needed with a larger selection of participants.

Therefore, this study aimed to explore the effect of PNF neck flexion exercise and the Shaker exercise on the activity of musculi suprahyoidei in chronic stroke patients with swallowing problems in a larger population sample. The purpose is to provide an effective exercise for swallowing rehabilitation based on the results of this study.

\section{Methods}

\section{Participant and Period}

This study was conducted at F hospital in Daegu from August 2014 to February 2016 in 60 participants who understood the purpose of the study and desired to participate. The selection criteria for the participants were as follows: participants who were diagnosed with stroke by CT or MRI, those who had been diagnosed with dysphagia after a stroke, those with an MMSE-K score of 24 or more, and those who did not have any other internal diseases other than stroke. This study was reviewed by the Institutional Review Board of Fatima Hospital in Daegu, Korea and was conducted with the consent of the participants and their guardians. The IRB review number is DFH14ORIO020.

\section{Experimental Method}

\section{1) Research procedure}

A total of 60 participants were randomly divided into an experimental group (PNF neck flexion exercise) $(n=30)$ 


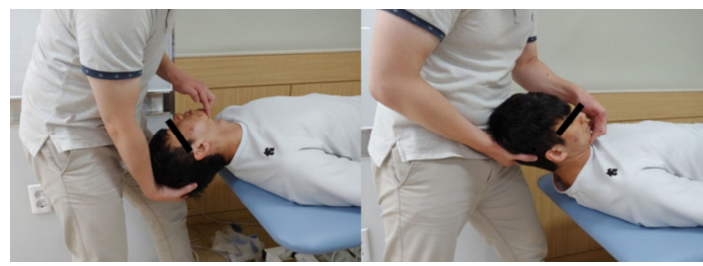

Fig. 1. PNF neck flexion exercise

and a control group (Shaker exercise) $(n=30)$. PNF neck flexion exercise was performed in the experimental group and the Shaker exercise was performed in the control group for 30 minutes, 5 times a week for 6 weeks. The activity of the suprahyoid muscles was measured before and after treatment.

\section{2) PNF neck flexion exercise}

In this study, PNF neck-flexion exercise was performed by the method suggested by Kim (2015) (Fig. 1). There are two types of PNF neck-flexion exercise. The first method is conducted in the order of flexion - right lateral flexion - right rotation and the second method is conducted in the order of flexion - left lateral flexion - left rotation.

The participant lay on the bed with the head and neck placed off the bed. The researcher stood on the left side behind the participant's head. The right hand of the experimenter supported the left occiput of the participant, and the fingertip of the left hand was positioned below the participant's chin. (The experimenter stood at the patient's head. One of the experimenter's hands was placed on the occiput and the fingertip of the other hand was placed under the patient's chin.) Before the exercise, the patient was given a detailed explanation on the direction of diagonal exercise. For the exercise starting posture, the patient's neck was pulled opposite of the exercise direction. At the start of the exercise, the patient was instructed to complete the chin movement first and then complete the flexion in order of lateral flexion followed by rotation. When the participant was suffering due to gravity, the experimenter helped the participant complete the exercise

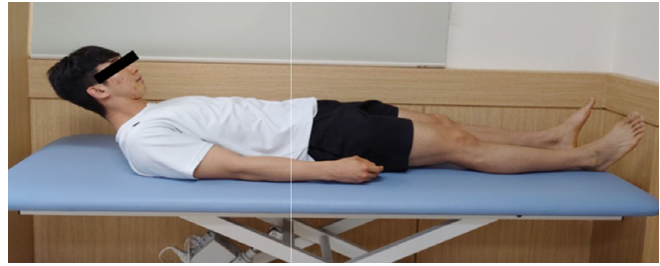

Fig. 2. The Shaker exercise

rather than using traction on their neck. Five sets of the exercise were performed and each set included 10 repetitions of the exercise. The participant was given enough time to relax between sets.

\section{3) The Shaker exercise}

The Shaker exercise is a head lift exercise developed by Shaker et al. (1997) (Fig. 2). This exercise consisted of 1 set of isometric and isotonic exercises. In the isometric exercise, the participant lay on his/her back and lifted his/her head to look at his/her toes for 60 seconds while avoiding raising his/her shoulders. In the isotonic Shaker exercise, the participant lifted his/her head to the same position and looked at his/her tiptoe 30 times in a row. If feeling exhausted, the patient was given a break in the middle of the exercise. The Shaker exercise was conducted under the supervision of the researcher so that the participant would exercise with the correct posture.

\section{4) Measurement equipment}

To measure the activity of the suprahyoid muscles, surface EMG (Biopac systems, Inc., Goleta, CA, USA) was used. To measure the muscle activity, \%RVC method was used. When the patient was sitting on a chair in stable condition for 3 seconds, the muscle activity in the suprahyoid muscle was measured while swallowing $2 \mathrm{ml}$ of water (Mun, 2013). The measurement posture was as follows: The participant was seated with correct posture on a chair with a backrest. The height of the chair was set to the knee height of the patient. Electrodes were attached on both sides of the centerline under the chin, 


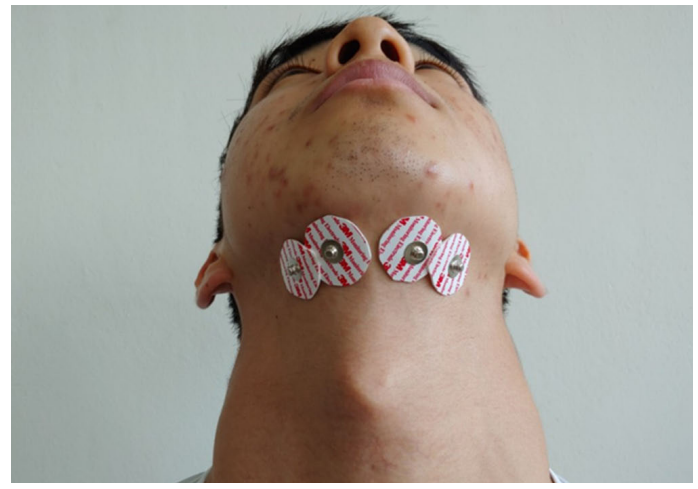

Fig. 3. Position of electrodes

$1 \mathrm{~cm}$ apart (Valenzuela et al., 2006) (Fig. 3). Furthermore, hair was removed to prevent measurement error and the electrodes were attached after wiping with medical alcohol. The sampling rate for the EMG signal was set to 1024 Hz. After the measurements, data were filtered and processed using Biopac Student Lab Pro (Biopac System Inc. CA. USA) on a personal computer. In order to remove noise within the measured EMG signal, band pass filtering was conducted on the raw data and smoothing was also performed as RMS $20 \mathrm{~ms}$ after rectification (Lee, 2014).

\section{5) Statistical analysis}

SPSS Window 18.0 was used to analyze the study results, and the results obtained were reported as the mean and standard deviation. Descriptive statistics were used to describe the general characteristics of the participants and the paired t-test was conducted to compare the suprahyoid muscle activity of the experimental group and the control group before and after the treatment. An independent t-test was performed to compare the changes in activity of the suprahyoid muscles by group. All statistical significance levels (a) were set below .05.

\section{Results}

1. General characteristics of study participants

The general characteristics of the study participants are described in Table 1. The study participants had no statistically significant characteristics.

\section{Intrarater reliability of activity in muscle}

Muscular activity of musculi suprahyoidei was measured three times to measure intrarater reliability. The reliability of musculi suprahyoidei upon PNF neck bending exercise was as high as .85 at minimum and .97 at maximum (Table 2).

Table 1. General Characteristics of the Two Groups

\begin{tabular}{ccccc}
\hline & PEG group (n=30) & SEG group (n=30) & $t$ & $p$ \\
\hline Sex (male/female, n) & $15 / 15$ & $15 / 15$ & & \\
Age (year) & $60.13 \pm 8.98$ & $62.43 \pm 6.87$ & -1.114 & .270 \\
Height (cm) & $161.00 \pm 8.46$ & $160.33 \pm 8.32$ & .307 & .760 \\
Weight (kg) & $63.56 \pm 11.37$ & $65.06 \pm 10.42$ & -.532 & .597 \\
Duration (month) & $7.86 \pm 4.18$ & $7.43 \pm 3.82$ & .418 & .677 \\
MMSE-K & $27.20 \pm 2.09$ & $27.53 \pm 2.08$ & -.619 & .538 \\
Type of stroke (infarction/hemorrhage, n) & $14 / 16$ & $15 / 15$ & $19 / 11$ & \\
Paretic side (right/left, n) & $17 / 13$ & & \\
\hline
\end{tabular}

$* * * \mathrm{P}<.001, * * \mathrm{P}<.01, * \mathrm{P}<.05$

$\mathrm{M} \pm \mathrm{SD}:$ Mean \pm Standard Deviation

PEG : PNF neck flexion exercise group, SEG : Shaker exercise group 
Table 2. Intrarater Reliability of Activity in Muscle

\begin{tabular}{cccc}
\hline Group & Muscle & ICC & $95 \%$ CI \\
\hline PEG & Suprahyoid & .85 & $.75 \sim .92$ \\
SEG & Suprahyoid & .97 & $.94 \sim .98$ \\
\hline
\end{tabular}

ICC : intraclass correlation coefficient

$\mathrm{CI}$ : confidence interval

PEG : PNF neck flexion exercise group, SEG : Shaker exercise group

Table 3. Comparison of Suprahyoid Muscle \%RVC in Each Groups (\%RVC)

\begin{tabular}{cccccc}
\hline Group & Pre-test & Post-test & Difference value & $t$ & $p$ \\
\hline PEG $(\mathrm{mV})$ & $214.93 \pm 56.65$ & $493.60 \pm 105.80$ & $278.66 \pm 113.56$ & -13.441 & $.000^{* * *}$ \\
SEG $(\mathrm{mV})$ & $223.23 \pm 53.75$ & $371.53 \pm 92.15$ & $148.30 \pm 88.13$ & -9.217 & $.000^{* * *}$ \\
$t$ & & 4.967 & & \\
$p$ & & & $.000^{* * *}$ & \\
\hline
\end{tabular}

$* * * \mathrm{P}<.001, * * \mathrm{P}<.01, * \mathrm{P}<.05$

$\mathrm{M} \pm \mathrm{SD}$ : Mean \pm Standard Deviation

PEG : PNF neck flexion exercise group, SEG : Shaker exercise group

\section{Comparison of Suprahyoid Muscle Activity} within Groups

For the PNF group, the suprahyoid muscle activity was $214.93 \pm 56.65$ before intervention and increased to $493.60 \pm 105.80$ after intervention. For the Shaker group, the suprahyoid muscle activity was $223.23 \pm 53.75$ before intervention and increased to $371.53 \pm 92.15$ after intervention. Both groups showed a significant change (Table 3).

\section{Comparison of Suprahyoid Muscle Activity between Groups}

After the intervention, the PNF group showed a change of 278.66 \pm 113.56 (\% RVC) and the Shaker (control) group showed a change of $148.30 \pm 88.13$ ( $\%$ RVC). The PNF group had a greater change than the Shaker group and the changes were statistically significant when compared by group (Table 3 ).

\section{Discussion}

In swallowing, the pharyngeal phase starts from strong contraction of musculi suprahyoidei along with the swallowing reflex. This moves the hyoid bone to the front, directly affecting the opening of the upper esophageal sphincter (Kim and McCullough, 2008). Weakening of the musculi suprahyoidei reduces the movement of the hyoid bone, affecting the time of food passage and causing suction by reducing the opening of the upper esophageal sphincter. This can cause severe dehydration, malnutrition, pneumonia and death (Bingiie et al., 2010). In rehabilitation of swallowing problems, alongside neuroplastic changes, the muscles under the chin must be strengthened (Won, 2012). Yoon et al. (2014) and Watts (2013) reported that effective resistive exercise will prevent aspiration at the pharynx due to muscular activation of musculi suprahyoidei.

Mepani et al. (2009) compared a Shaker exercise group with a traditional swallowing treatment group and reported that the Shaker exercise improves the muscular power of musculi suprahyoidei. Mun (2013) showed a change of about $200 \% \mathrm{RVC}$ in the muscle activities of musculi suprahyoidei before and after the Shaker exercise, while 
Kim (2015) reported a change of about 90\% RVC and the current study found a change of about $148 \% \mathrm{RVC}$. Mun (2013) reported the greatest change because 16 out of 20 patients in that study were sub-acute patients. The study by Kim (2015) included chronic stroke patients only. However, the sample size was small, resulting in the difference compared to this study. As demonstrated by these studies, the Shaker exercise has a great effect on activation of the musculi suprahyoidei.

According to recent studies, resistive neck bending exercise, rather than the Shaker exercise, helps to strengthen musculi suprahyoidei and swallowing (You, 2016). Yoon et al. (2014) reported chin tuck against resistance (CTAR) exercise resulted in higher muscular activity of musculi suprahyoidei than the Shaker exercise. Watts (2013) reported that the muscular activity of muscles under the chin was higher in chin-opening exercise against resistance than in the Shaker exercise. Also, there are recent, continuous studies on the effect of PNF neck flexion exercise on the muscular activity of musculi suprahyoidei and swallowing function. Lee and Won (2015) presented a case study in which PNF neck flexion exercise improved swallowing function. Kim (2015) also reported that PNF neck flexion exercise improved the muscular activity of musculi suprahyoidei and swallowing function and that the effects were similar to those of the Shaker exercise. In this study, PNF neck flexion exercise resulted in a change of about $278 \%$ RVC. This change was greater than that of the Shaker exercise (148\%RVC) and was statistically significant.

These results indicate that PNF neck flexion exercise increased the muscular activity of musculi suprahyoidei more than the Shaker exercise. Also, this increase in musculi suprahyoidei activity increases movement of the hyoid bone and decreases aspiration, helping with swallowing.

PNF neck flexion exercise has some disadvantages. First, treatment should occur 1:1 between a patient and a therapist.
Second, therapists must have the skills required to safely use PNF patterns. Still, despite these few disadvantages, the effect on muscular activity of musculi suprahyoidei is high. PNF neck flexion exercise also has an advantage that it can be conducted in many positions such as lying, sitting and side-lying. In addition, neck bending muscular power can be used instead of resistance to complete the exercise in patients with only fair strength or below. Based on the results, it is recommended that PNF neck flexion exercise be applied clinically according to the state and treatment status of the patient.

Considering that the sample size in the study by Kim (2015) was small, the author invited 60 chronic stroke patients with dysphagia. However, this study only measured the activity of musculi suprahyoidei of patients with a swallowing disability. This study was limited in that it did not measure substantial swallowing functions. In the future, both the musculi suprahyoidei activity and swallowing should be measured to study the effects of PNF neck flexion exercise and the Shaker exercise. Also, there should be a study to determine how increasing the musculi suprahyoidei activity affects the speed of swallowing in patients with a swallow disability.

\section{Conclusion}

This study was conducted to investigate the effect of PNF neck flexion and the Shaker exercise on the activity of the suprahyoid muscle in chronic stroke patients with dysphagia. The activity of the suprahyoid muscles was statistically significantly different before and after treatment in the experimental group and the control group. Statistical significance was also found in comparing the two groups. Based on the results, both PNF neck flexion exercise and the Shaker exercise effectively activate the musculi suprahyoidei. However, when comparing PNF neck flexion exercise with the Shaker exercise, the former was more effective than the latter. 
In conclusion, PNF neck flexion exercise should be applied in addition to the Shaker exercise when rehabilitating a patient with a swallow disability.

\section{Acknowledgements}

This research was carried out in 2018 with the support of the research fund of Dongju College.

\section{References}

Bingie L, Tong Z, Xinting S, et al. Quantitative videofluoroscopic analysis of penetration-aspiration in post-stroke patients. Neurology India. 2010; 58(1):42-7.

Choi JB, Shim SH, Yang JE, et al. Effects of Shaker exercise in stroke survivors with oropharyngeal dysphagia. Neurorehabilitation. 2017;41(4):753-7.

Easterling C, Kern M, Nitschke T, et al. Restoration of oral feeding in 17 tube fed patients by the Shaker Exercise. Dysphagia. 2000;15(2):105-9.

Hong DK, Kyoung KS, Yu DH. The effect of Shaker exercise on swallowing function of stroke patients. Journal of Korean Society of Occupational Therapy. 2012; 20(3):55-66.

Kim KD. The effect of PNF neck-flexion exercise on swallowing function of chronic stroke patients. Doctor's Degree. Daegu university. 2015.

Kim Y, McCullough GH. Maximum hyoid displacement in normal swallowing. Dysphagia. 2008;12(3):274-9.

Kofotolis N, Kellis E. Effects of two 4-week proprioceptive neuromuscular facilitation programs on muscle endurance, flexibility, and functional performance in women with chronic low back pain. Physical Therapy. 2006;86(7):1001-12.

Lee MH. The effect of cervical stabilization exercise on respiration function of stroke patients. Doctor's Degree. Daegu university. 2014.

Lee SH, Won YS. The effect of neck strengthening exercise using proprioceptive neuromuscular facilitation on swallow ability of patient with dysphagia : A single case study. Journal of the Korean Proprioceptive Neuromuscular Facilitation Association. 2015;13(3): 163-8.

Logemann JA, Rademaker A, Pauloski BR, et al. A randomized study comparing the Shaker exercise with traditional therapy: a preliminary study. Dysphagia. 2009;24(4): 403-11.

Mepani R, Antonik S, Massey B, et al. Augmentation of deglutitive thyrohyoid muscle shortening by the Shaker exercise. Dysphagia. 2009;24(1):26-31.

Mun TH. The effects of shaker exercise and neuromuscular electrical stimulation therapy on the swallowing function: on the post-stroke patients with dysphasia. Master's Degree. Inje university. 2013.

Oh JC. Effects of compensatory swallowing strategies on submental muscle activation. Korea Academy of Dysphagia Rehabilitation. 2018;1(1):1-6.

Shaker R, Easterling C, Kern M, et al. Rehabilitation of swallowing by exercise in tube-fed patients with pharyngeal dysphagia secondary to abnormal UES opening. Gastroenterology. 2002;122(5):1314-21.

Shaker R, Kern M, Bardan E, et al. Augmentation of deglutitive upper esophageal sphincter opening in the elderly by exercise. American Journal of Physiology. 1997;272(6):1518-22.

Singh S, Hamdy S. Dysphagia in stroke patients. Postgraduate Medical Journal. 2006;82(968):383-91.

Adler SS, Beckers D, Buck M, et al. PNF in Practice. Springer. 2000

Valenzuela S, Baeza M, Miralles R, et al. Laterotrusive occulusal schemes and their effect on supra- and infrahyoid electromyographic activity. The Angel Orthodontist. 2006;76(4):585-90.

Watts CR. Measurement of hyolaryngeal muscle activation using surface electromyography for comparision of two rehabilitative dysphagia exercise. Archives of 
Physical Medicine and Rehabilitation. 2013;94(12): 2542-8.

White KT, Easterling C, Roberts N, et al. Fatigue analysis before and after shaker exercise: physiologic tool for exercise design. Dysphagia. 2008;23(4):385-91.

Won YS. The effects of oropharyngeal exercise combined with tongue pressure training protocol on swallowing function in stroke patients with dysphagia. Journal of Special Educaton and Rehabilitation Science. 2012;51(2):57-71.
Woo HS, Park SH, Jung MY, et al. The effects of craniocervical flexion on activation of swallowingrelated muscles. Journal of Oral Rehabilitation. 2012;39(11):805-11.

Yoon WL, Khoo JKP, Liow SJR. Chin tuck against resistance (CTAR): new method for enhancing suprahyoid muscle activity using a Shaker-type exercise. Dysphagia. 2014;29(2):243-8.

You SJ. Effects of Shaker and chin tuck against resistance (CTAR) exercise on dysphagia according to muscle activity and fatigue in healthy adults. Doctor's Degree. Daegu university. 2016. 